\title{
Revealing trap depth distributions in persistent phosphors
}

\author{
Koen Van den Eeckhout, ${ }^{1,2}$ Adrie J. J. Bos, ${ }^{3}$ Dirk Poelman,,${ }^{1,2}$ and Philippe F. Smet ${ }^{1,2}$ \\ ${ }^{1}$ LumiLab, Department of Solid State Sciences, Ghent University, Belgium \\ ${ }^{2}$ Center for Nano- and Biophotonics (NB-Photonics), Ghent University, Belgium \\ ${ }^{3}$ Faculty of Applied Sciences, Delft University of Technology, The Netherlands
}

(Received 21 December 2012; revised manuscript received 15 January 2013; published 28 January 2013)

\begin{abstract}
Persistent luminescence or afterglow is caused by a gradual release of charge carriers from trapping centers. The energy needed to release these charge carriers is determined by the trap depths. Knowledge of these trap depths is therefore crucial in the understanding of the persistent luminescence mechanism. Unfortunately, the trap depths in persistent phosphors are often difficult to evaluate in an accurate and reliable way. The existing analysis methods are mostly based on single experiments, or they ignore the possibility of a continuous distribution of trap depths. We present a procedure to accurately probe the activation energies, even in the presence of a continuous distribution of energy levels. By performing a series of thermoluminescence experiments with varying excitation duration and at varying excitation temperature, and employing the initial rise analysis method, the depth and shape of such a distribution can be estimated. As an example, we investigated the trap system in the violet persistent phosphor $\mathrm{CaAl}_{2} \mathrm{O}_{4}: \mathrm{Eu}, \mathrm{Nd}$, and show that it consists of a Gaussian-shaped distribution of trap depths. The maximal density of traps lies in the region around $0.9 \mathrm{eV}$, but the distribution extends to $0.7 \mathrm{eV}$ on the shallow side and $1.2 \mathrm{eV}$ on the deep side. The described procedure can be used to obtain a clear view of the trap system in other persistent phosphors as well. This can lead to a better understanding of the nature of these trapping centers, and the role they play in the persistent luminescent mechanism.
\end{abstract}

\section{INTRODUCTION}

Contrary to most luminescent materials, where the light emission lasts only milliseconds after the end of the excitation, persistent luminescent materials can continue emitting light for minutes or hours. This remarkable property has been known since ancient times, ${ }^{1}$ but research into persistent luminescence has seen a dramatic increase since the discovery of the bright and long-lasting green afterglow of $\mathrm{SrAl}_{2} \mathrm{O}_{4}$ : Eu,Dy in 1995 by Matsuzawa et $a .^{2}$ Unfortunately, despite this intensive search, only a handful of other efficient persistent phosphors have been developed since then. The most notable of these are $\mathrm{Sr}_{2} \mathrm{MgSi}_{2} \mathrm{O}_{7}: \mathrm{Eu}, \mathrm{Dy}\left(\right.$ blue $\left.^{3}\right), \mathrm{Sr}_{4} \mathrm{Al}_{14} \mathrm{O}_{25}$ : Eu,Dy (green ${ }^{4}$ ), $\mathrm{Y}_{2} \mathrm{O}_{2} \mathrm{~S}: \mathrm{Eu}, \mathrm{Ti}, \mathrm{Mg}\left(\right.$ (red $^{5}$ ), and $\mathrm{CaAl}_{2} \mathrm{O}_{4}: \mathrm{Eu}, \mathrm{Nd}$ (violet ${ }^{6}$ ).

One of the main reasons for this relative scarcity of bright afterglow phosphors is the trial-and-error nature of the persistent luminescence research. Although most of the general principles behind the afterglow mechanism are now understood, many of the details remain the subject of discussion. ${ }^{7}$ A crucial role is played by energy levels in the band gap of the host material, introduced by defects in the crystal lattice (e.g., vacancies or codopants). These so-called "traps" are able to capture charge carriers originating from the luminescent centers (electrons in most cases, ${ }^{8}$ although hole trapping has been suggested by some authors and for some materials ${ }^{7}$ ). These charge carriers remain trapped until enough thermal energy is available to help them escape and recombine at a luminescent center.

The activation energy required for this is called the trap depth and is supposedly determined by the energy difference between the energy level of the trap and the conduction band (in the case of electron trapping) or the valence band (in the case of hole trapping). A trap which is too shallow (i.e., too close to the conduction-or valence-band) will result in a very short afterglow; if the trap is too deep no charge carriers can escape at room temperature and no persistent luminescence will be observed unless the temperature is raised. A trap depth of around $0.6-0.7 \mathrm{eV}$ is often stated as ideal for persistent luminescence. ${ }^{2}$

Clearly, knowing the number and depth of these energy levels is crucial when trying to understand the mechanism of persistent luminescence, and when developing new afterglow materials. Unfortunately, the trap system often consists of multiple levels with similar depths, or even continuous distributions of energy levels. This complicates an accurate determination of the traps in the phosphor under investigation, let alone the possible interactions between them.

We present a procedure to extract information on the trap system in persistent phosphors in a straightforward and physically intuitive way. This procedure is described in Sec. II. By performing a series of thermoluminescence (TL) experiments with varying excitation duration and at varying excitation temperature, the presence of a continuous trap depth distribution-rather than one or more discrete energy levels — can be verified. Furthermore, by employing the initial rise analysis method it is possible to estimate the depth and shape of this distribution. As an example, this procedure is applied in Sec. III to the well-known violet persistent phosphor $\mathrm{CaAl}_{2} \mathrm{O}_{4}: \mathrm{Eu}, \mathrm{Nd}$.

The same procedure can be applied to storage phosphors and scintillators, where knowledge of the trap depths is of equally crucial importance. ${ }^{9}$

\section{PROBING TRAP DEPTH DISTRIBUTIONS}

During the past decades, several methods for estimating trap depths in persistent phosphors have been developed. ${ }^{10,11}$ Most of these start from glow curves obtained through thermoluminescence experiments. Roughly, they can be divided into five broad categories: (1) initial rise methods, based on 
the initial fraction of the glow curve; (2) area methods, based on the area under the glow curve; (3) peak position methods, based on the location of the maximum; (4) shape methods, based on the shape and symmetry of the curve; and (5) curve fitting methods, computerized or using a series expansion.

The best known and most used of these are the Hoogenstraaten method, ${ }^{12}$ based on the shift of the peak position under variable heating rates, the shape method developed by Chen, ${ }^{13}$ and the computerized fitting of the glow curve using specialized software. ${ }^{14,15}$

Each of these methods has its own shortcomings. Often, the presence of discrete trap depths is assumed, and the possibility of a continuous trap depth distribution is not considered. Furthermore, many of these methods are applied to the glow curve acquired during a single TL measurement. This makes the interpretation of the obtained results physically less sound.

In the following paragraphs, we describe a procedure to more accurately probe the trap system in persistent phosphors, even in the case of a continuous distribution of trap depths. This procedure is based on two principles: (a) performing a series of TL experiments with varying excitation duration and varying excitation temperature and (b) analyzing these TL experiments using the initial rise method.

The advantage of such a procedure is twofold. By performing a series of TL experiments, the obtained result will be more reliable and we will be able to prove the presence of a continuous trap depth distribution. Secondly, the initial rise analysis provides a good estimate of the trap depth, independent of the trapping kinetics, even when such a continuous trap depth distribution is present.

\section{A. Thermoluminescence}

TL is a powerful and versatile tool to investigate the depth of the trap(s) present in persistent phosphors. It is frequently used in storage phosphors for dosimetry and geological dating purposes, ${ }^{10}$ but is also becoming increasingly common in persistent luminescence research.

The principle is as follows. First, the material of interest is heated to ensure that all the charge carrier traps are empty. Subsequently, the sample is excited for a certain time using $\mathrm{UV}$ radiation (or $\mathrm{x}$-ray radiation in the case of scintillators or storage phosphors) at low temperature (commonly room temperature, but liquid nitrogen or helium temperature are also used). The material is then heated in a controlled way, usually with a constant heating rate, while the light output is continuously measured.

The appearance of a peak in the emitted light intensity (glow peak) indicates the presence of a charge carrier trap in the material. The temperature at which this peak is located is a measure for the trap depth, since it is the temperature at which enough thermal energy is available for the trapped charge carriers to be released and recombine at luminescent centers. The shape, height, and location of the peak all contain information on the number and depth of the traps and the kinetics of the trapping and detrapping process.

\section{First, second, and general order kinetics}

In the case of first order kinetics, described by Randall and Wilkins in $1941,{ }^{16}$ the possibility of retrapping is assumed to be negligible. In other words, every charge carrier that manages to escape a trap immediately produces luminescence and is not captured by another trap. This leads to an exponential afterglow decay profile and an asymmetric TL glow peak with a negative skew.

Randall and Wilkins already noted the possibility that retrapping does play an important role during TL measurements. ${ }^{17}$ In 1948, Garlick and Gibson derived expressions for the case of an equal probability for both processes, ${ }^{18}$ known as "second order kinetics." The afterglow decay profile in this case no longer follows an exponential function, but can be described by a $(1+t)^{-2}$ behavior. For the limit $t \rightarrow \infty$, the afterglow follows a power law with a power of -2 . The $\mathrm{TL}$ peak in this case is more symmetrical compared to the first order case.

The expressions for first and second order kinetics are only valid in ideal situations, i.e., when the retrapping probability is negligible or when it is equal to the recombination probability. To describe realistic situations in between these two extremes, May and Partridge introduced an empirical relation in 1964 denoted as "general order kinetics."19 They derived expressions which are nowadays commonly employed, especially since the introduction and profusion of software for TL glow curve fitting. However, it must be kept in mind that the general order approach is a purely mathematical interpolation between the simplified cases of first and second order kinetics, and therefore lacks a straightforward physical meaning.

Figure 1 summarizes how the order of kinetics influences the shape of a glow peak. In the case of first order kinetics $(b=1)$, the peak is asymmetric with a strongly negative skew, while for second order kinetics $(b=2)$ the peak is almost symmetric. For general order kinetics, the curve lies in between these two extremes.

\section{Continuous trap depth distributions}

Most afterglow decay curves, as well as glow curves obtained in a TL experiment, cannot be explained by the simplest model of a single discrete energy level with first,

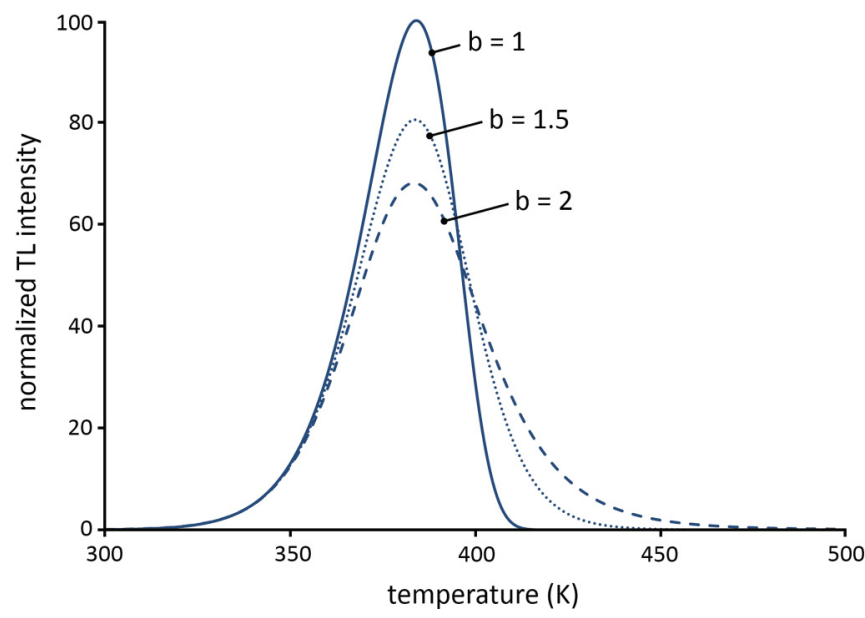

FIG. 1. (Color online) Typical glow curve shape for first, second, and general order kinetics. $E=1 \mathrm{eV}, n_{0}=N=1 \mathrm{~m}^{-3}, s=1 \times$ $10^{12} \mathrm{~s}^{-1}$, and $\beta=1 \mathrm{~K} / \mathrm{s}$. (Adapted from Ref. 20.) 
second, or even general order kinetics. Afterglow decay curves often follow a $t^{-1}$ behavior, ${ }^{21}$ which cannot be understood by any of these models. The same is true for many TL glow peaks, which are often too broad-especially on the high-temperature side - to be described by a single first, second, or general order glow peak.

A common approach is therefore to assume the presence of multiple discrete energy levels obeying first order kinetics. The decay curve is then decomposed into multiple (three or more) exponential components, ${ }^{22-25}$ frequently called "fast," "slow," or "intermediate," however, the time constants obtained in this way are often difficult to interpret physically and the large number of parameters involved can make the fitting procedure unreliable.

Similarly, broad TL glow peaks are often fitted to three or more individual glow peaks obeying general order kinetics. Again, since each glow peak has five independent parameters (the trap depth $E_{T}$, the frequency factor $s$, the trap concentration $N$, the initial concentration of filled traps $n_{0}$, and the order $b$ ) 15 or more parameters are needed for fitting, making a physical interpretation impossible. This is especially the case if only a single TL experiment is taken into account, as opposed to a series of experiments performed on a single sample.

Another approach to explain the broadness of the TL peaks is assuming the presence of a trap depth distribution. In this case, the trap level is not regarded as a discrete energy level located in the band gap of the host material, but rather a continuum of energy levels around a certain mean value. This possibility has been explored by various authors, ${ }^{21,26-41}$ and three main types of trap distributions have been considered: uniform, exponential, and Gaussian profiles.

The advantage of this approach is twofold. First, a distribution of trap depths requires fewer parameters to be accurately described than a multitude of discrete energy levels. Secondly, it is believed that a continuous distribution of trap depths is a more intuitively acceptable description of the actual defect situation in inorganic phosphors since the structure of the lattice surrounding the trapping center may exhibit random variations in the nearest neighbor bond angles and bond lengths. Also, association of the trapping centers with other defects in the vicinity is possible. In this case, small variations in the spatial configurations of the defect aggregates can cause a broadening of the trap depth distribution. Another possible origin for variations in trap depths is the distance from the traps to the luminescent centers, as has been shown in, e.g., $\mathrm{Lu}_{x} \mathrm{Y}_{2-x} \mathrm{SiO}_{5} \cdot{ }^{42}$

The presence of such a continuous trap distribution directly influences the shape of the TL glow peaks by broadening them, and can also explain the observed $t^{-1}$ decay behavior of the afterglow, ${ }^{31}$ although other authors suggest this behavior could be due to a trapping mechanism governed by tunneling. ${ }^{43}$ In principle, these two explanations could be experimentally distinguished, since the $t^{-1}$ behavior in the case of a continuous trap depth distribution is dependent on the temperature (at different temperatures greater or smaller parts of the distribution are filled), while tunneling is temperature independent. However, such an experimental verification is beyond the scope of this text.
The first order kinetics formula ${ }^{16}$ can be modified to incorporate such a continuous distribution of traps by integrating over the trap depth: ${ }^{10}$

$$
\begin{aligned}
I(T)= & \int_{0}^{\infty} \frac{s}{\beta} N\left(E_{T}\right) f_{0}\left(E_{T}\right) \exp \left(-\frac{E_{T}}{k T}\right) \\
& \times \exp \left[-\frac{s}{\beta} \int_{T_{0}}^{T} \exp \left(-\frac{E_{T}}{k \theta}\right) d \theta\right] d E_{T},
\end{aligned}
$$

where $s$ is the frequency factor, $\beta$ is the heating rate, $E_{T}$ is the trap depth, $N\left(E_{T}\right)$ is the distribution of available trap depths (this can be uniform, exponential, Gaussian,...), and $f_{0}\left(E_{T}\right)$ describes which fraction of the traps is filled at $t=0 . k$ is the Boltzmann constant.

It should be noted that integrating over the energy range of the distribution is only valid for first order kinetics. In the case of higher order kinetics, the presence of retrapping complicates the process, since the escaped charge carriers can be captured by neighboring traps with different activation energies than the one they escaped from. This interaction between trap levels with various trap depths makes the simple integration over the energy range invalid.

For practical purposes, this glow curve behavior in the presence of a trap depth distribution can be simulated numerically. For this, it suffices to divide the continuous distribution into a large number of small intervals, calculate the glow curve for each of these intervals, and add these glow curves to obtain the curve for the entire trap distribution. It was shown by Rudlof et al. ${ }^{44}$ and Hornyak and Chen, ${ }^{21}$ that the results of such a numerical approach agree very well with the analytical expression.

\section{Series of TL experiments}

Compared to a single glow curve, a series of TL experiments on the same sample yields a lot more information than a single experiment. First, more data is available to analyze, making the final result more accurate and more reliable. Secondly, performing a series of subsequent TL experiments while changing only a single parameter between each measurement can yield additional information which cannot be extracted from only one glow peak.

To study trap depth distributions in persistent phosphors, two parameters are particularly interesting: the duration of the excitation and the temperature during the excitation of the sample. Varying the excitation duration provides insight into the kinetics of the trapping and detrapping process. Indeed, in the case of first order kinetics we expect the location of the peak maximum to be independent of the excitation dose (and hence the excitation duration), while for higher order kinetics a shift of the maximum is expected. ${ }^{20}$

To study the presence of a trap depth distribution it is interesting to vary the temperature at which the sample is excited. The reason for this is graphically represented in Fig. 2. If a phosphor with a continuous trap depth distribution is excited at a higher temperature, only deeper fractions of the distribution are filled. The shallower traps are immediately bleached because of the increased thermal energy available. If we then estimate the trap depth, the resulting value will increase with increasing excitation temperature. 


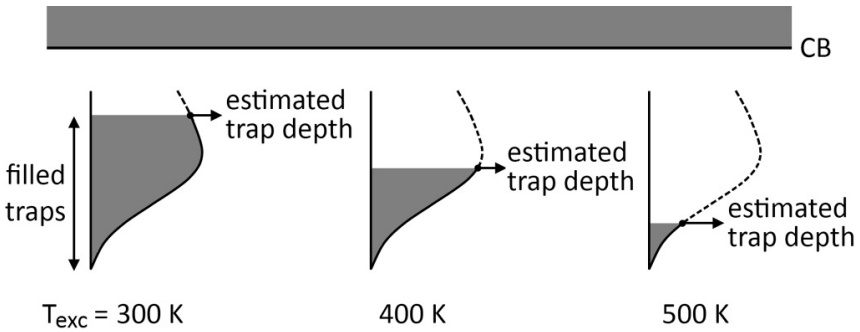

FIG. 2. Schematic representation of the fractions of a trap distribution that are filled, as a function of increasing excitation temperatures.

\section{B. Initial rise analysis}

The initial rise approach for estimating trap depths starts from the assumption that on the low-temperature side of a TL glow curve, the concentration of trapped charge carriers is relatively constant. Only a tiny fraction of the charge carriers can escape given the small amount of thermal energy available. We can therefore approximate the equations for first, second, and general order kinetics to

$$
I(T)=C \exp \left(\frac{-E_{T}}{k T}\right) .
$$

The constant $C$ includes the frequency factor $s$ which is assumed to be independent of the temperature. If we now plot the glow curve as $\ln (I)$ versus $1 / T$, i.e., in an Arrhenius plot, the low-temperature side of the peak will resemble a straight line, from which the trap depth can be readily estimated. This is illustrated in Fig. 3, which shows a simulated glow curve for a uniform trap distribution stretching from 0.9 to $1.1 \mathrm{eV}$, assuming first order kinetics and $\beta=5 \mathrm{~K} / \mathrm{s}$. Note that even in the case of a relatively broad continuous distribution a straight section in the Arrhenius plot can be clearly resolved. For an accurate result, typically only the fraction where $I<0.15 I_{\max }$ should be considered. ${ }^{11,45}$

The initial rise method has two major advantages. First, it is independent of the order of kinetics involved in the trapping and detrapping processes. Secondly, since only a part of the glow peak is considered (the low-temperature side), the problem of overlapping TL peaks is greatly reduced. However, for two largely overlapping peaks, the initial part of the highest-temperature peak might be obscured by the tail of (a)

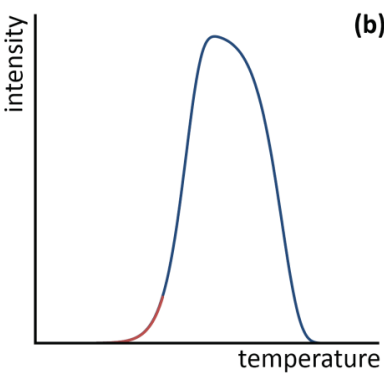

(b)

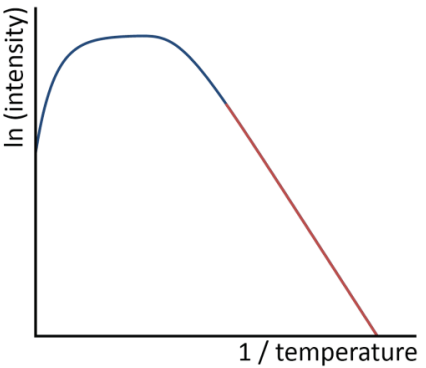

FIG. 3. (Color online) The initial rise analysis for estimating trap depths, illustrated for a simulated glow curve of a continuous trap depth distribution. (a) TL glow curve. (b) Glow curve in an Arrhenius diagram. The slope of the straight section on the low-temperature side is determined only by the trap depth: slope $=-E_{T} / k$.
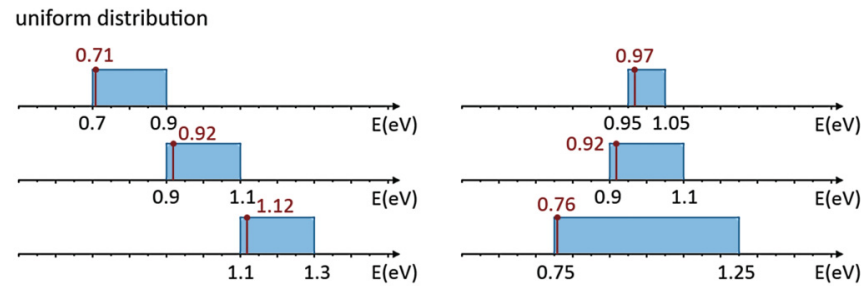

gaussian distribution
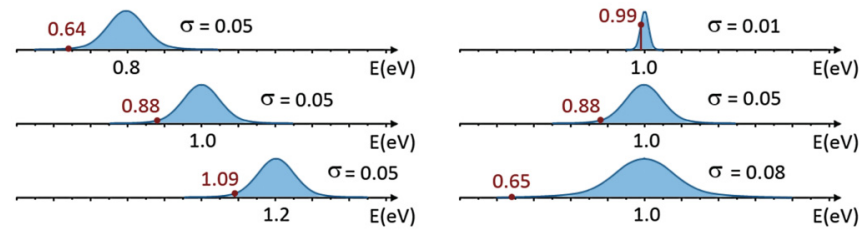

FIG. 4. (Color online) Simulation of trap depth estimations (indicated in red, in eV) using the initial rise method, for various (artificial) uniform and Gaussian distributions (indicated in blue).

the low-temperature peak. In this case, it is possible that no straight section can be resolved in the Arrhenius plot, and the initial rise analysis cannot be employed. Thermal cleaning of the traps ${ }^{46}$ might partially resolve this problem.

In the case of a continuous trap depth distribution, the initial rise analysis is expected to yield the depth of the shallowest occupied traps in the distribution. Indeed, we look only at the low-temperature region of the glow peak, where only the charge carriers in the shallowest traps are involved. The deeper traps remain filled because not enough thermal energy is available for the charge carriers to escape.

This expectation can be verified by simulations. In a first step, a trap depth distribution is created and the glow curve is simulated using the procedure described in the final paragraph of Sec. II A2, by dividing the continuous distribution into a large number of discrete intervals. Secondly, this simulated glow curve is analyzed using the initial rise procedure and the obtained estimation for the trap depth is compared to the actual trap distribution used. Such simulations were performed for both uniform and Gaussian trap depth distributions.

An overview of the results is presented in Fig. 4. The created trap depth distributions are indicated in blue, and the estimated trap depths are shown in red. As can be seen, the initial rise procedure always produces a value at the shallowest end of the distribution. For uniform distributions, the estimation is within $2.1 \%$ of the low energy edge. For a Gaussian distribution, the estimated value lies around the $E_{0}-3 \sigma$ mark, within a $3.9 \%$ error margin. Only for very broad distributions is the estimated trap depth somewhat lower, around the $E_{0}-4 \sigma$ mark $(0.65 \mathrm{eV}$ for a distribution around $1 \mathrm{eV}$ with $\sigma=0.08 \mathrm{eV}$ ).

From these results, we can conclude that the initial rise analysis indeed gives a good estimation for the trap depth of the shallowest occupied trap levels. No influence of the frequency factor $s$ or the heating rate $\beta$ was found.

\section{Combining TL and initial rise analysis for trap depth spectroscopy}

As discussed above, the presence of a trap depth distribution can be proven by performing a series of TL experiments at varying excitation temperatures and performing the initial rise 
analysis. If the estimated trap depth increases continuously with increasing excitation temperature, there is a strong indication for the presence of a continuous trap distribution, rather than one or more discrete energy levels. The obtained trap depth value is a good estimate for the shallowest edge of this distribution.

Information on the shape of the continuous trap depth distribution can also be obtained. In other words, it can be estimated how many trap levels are available at each depth for charge carriers to be trapped in. From a statistical point of view, a Gaussian distribution of traps is the most probable option. Sakurai et $a l .{ }^{34}$ found proof for an exponential trap profile in brown microcline, but later showed that this exponential profile was in fact only the deeper side of a Gaussian distribution. ${ }^{35}$

This information on the shape of the distribution can be obtained by integrating the glow curves for various excitation temperatures. The surface under the glow curve is a measure for the total number of luminescent ions returning to the ground state during the TL experiment, and hence for the total number of trapped charge carriers. As shown in Fig. 2, only the deeper fraction of the trap depth distribution-the trap depth deeper than the value estimated using the initial rise analysis-is filled. Therefore, at each excitation temperature, the integrated TL intensity is directly related to the total number of traps below the estimated trap depth. This means that the difference between the integrated TL intensities at two different excitation temperatures is a measure for the number of available traps between the depths estimated for these two measurements. In this way, the density of traps at various depths can be derived. This is shown schematically in Fig. 5.

The presented procedure is in a way comparable to the so-called "preheating" or "thermal cleaning" technique, in which the sample is heated to a temperature $T_{\text {stop }}$ after the excitation and before the TL experiment. ${ }^{40,46-48}$ In this way, the continuous distribution of trap depths is also emptied up to a certain depth, depending on $T_{\text {stop }}$. However, care should be taken that the preheating phase in each experiment has the same duration, to avoid the effects of fading.

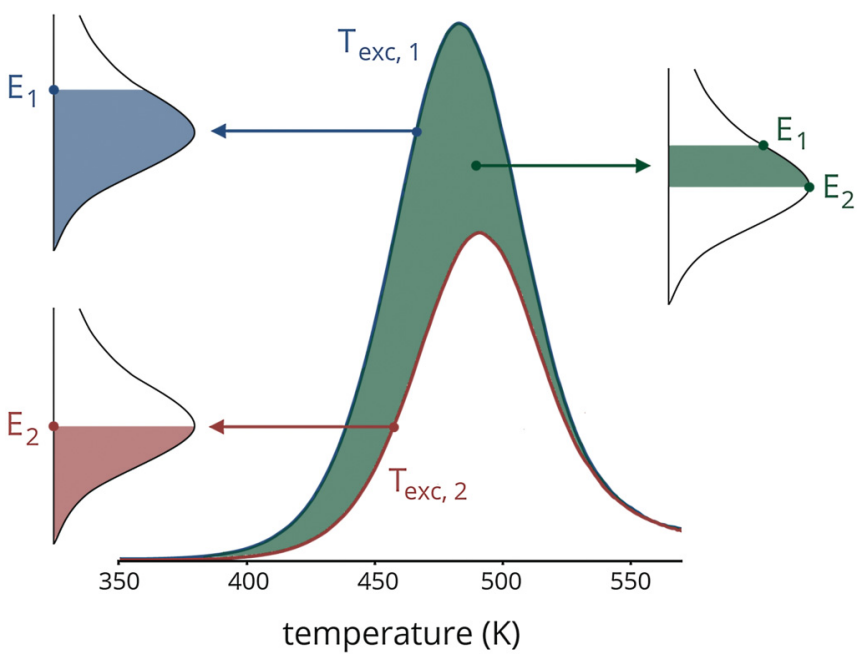

FIG. 5. (Color online) The density of the continuous trap depth distribution at a certain depth can be estimated from the difference between the integrated intensities at two different excitation temperatures.
An extension to the preheating technique is known as the "fractional glow" technique, in which the temperature is constantly varied during the TL measurement. ${ }^{27}$ Although this technique is also able to estimate the shape of the continuous trap depth distribution, the setup and data analysis are much more demanding. ${ }^{46}$

In principle, a fading experiment, where the duration between the excitation and the actual TL experiment is varied, could provide similar information about the presence of a trap depth distribution. However, for long fading times it is more difficult to accurately determine the trap depth because the edge between the filled and empty fraction of the trap distribution will be less clearly defined. Additionally, since the lifetime of the trapped charge carriers scales exponentially with the trap depth, the delay between the excitation and the TL measurement needs to be very long in order to study deeper parts of the distribution. For example, if we assume $s=10^{12} \mathrm{~s}^{-1}$, the average lifetime of a charge carrier in a trap with a depth of $E_{T}=1.1 \mathrm{eV}$ is nearly 150 days, which gives an idea of the measurement times required.

\section{TRAP DEPTH DISTRIBUTION IN $\mathrm{CaAl}_{2} \mathrm{O}_{4}$ :Eu,Nd}

As an illustration of the concepts explained above, the well-known persistent phosphor $\mathrm{CaAl}_{2} \mathrm{O}_{4}$ : $\mathrm{Eu}, \mathrm{Nd}$ was examined. This material, closely related to the aforementioned $\mathrm{SrAl}_{2} \mathrm{O}_{4}$ :Eu,Dy, has a long-lasting afterglow in the violet-blue region of the visible spectrum. Its luminescent properties were first described by Blasse and Bril ${ }^{49}$ and Palilla et al. ${ }^{50}$ in 1968, but in 1996 Matsuzawa et al. mentioned its persistent luminescence. $^{2}$ An afterglow duration of up to $10 \mathrm{~h}$ is mentioned in literature. $6,51,52$

The host material has a monoclinic crystal structure, ${ }^{53}$ but when prepared with a combustion or sol-gel method, a hexagonal or orthorhombic phase is sometimes obtained. ${ }^{54,55}$ The monoclinic structure has three possible cation sites, but the $\mathrm{Eu}^{2+}$ ions, which are somewhat larger than the $\mathrm{Ca}^{2+}$ ions, are only incorporated in the more spacious trigonal antiprism site. ${ }^{56}$ The presence of only a single europium site simplifies the analysis of the luminescence data (as compared to, e.g.. $\mathrm{SrAl}_{2} \mathrm{O}_{4}$ :Eu which has two possible europium sites ${ }^{56}$ ). For this reason, $\mathrm{CaAl}_{2} \mathrm{O}_{4}: \mathrm{Eu}$ is often chosen as a standard material for persistent luminescence investigations. ${ }^{41,52,57-63}$

\section{A. Experimental}

$\mathrm{CaAl}_{2} \mathrm{O}_{4}: \mathrm{Eu}, \mathrm{Nd}$ pellets were prepared via a nonaqueous sol-gel method ${ }^{57}$ and e-beam annealing as described in Ref. 64. The pellets produced using this procedure have an afterglow intensity of about three times that of commercially available powder, and the afterglow duration extends to $10 \mathrm{~h}$.

The emission and excitation spectra were measured in an Edinburgh FS920 fluorescence spectrometer with a 450W Xe arc light source. Afterglow decays were obtained using a calibrated photometer (ILT 1700, International Light Technologies) equipped with a photopic filter (YPM) and a Xe arc lamp for excitation at $1000 \mathrm{~lx}$. Thermal quenching was measured using a pulsed nitrogen laser $\left(\lambda_{\text {exc }}=337 \mathrm{~nm}\right.$, pulse length 800 ps) and a 1024-channel intensified CCD (Andor Technology) attached to a $0.5 \mathrm{~m}$ Ebert monochromator, in 
combination with a helium contact gas cryostat (Optistat, Oxford Instruments).

Thermoluminescent glow curves were obtained at Delft University of Technology, The Netherlands. The samples were exposed to $365 \mathrm{~nm}$ radiation at various temperatures and for various periods of time. The emission during heating was monitored using a Ris $\emptyset$ Thermoluminescence reader (TL/OSL-DA-15) collecting the glow curves by means of a photomultiplier tube and suitable optical filters. The setup is fully automated and software controlled by a user interface written in LABVIEW. ${ }^{65}$ For experiments with an excitation temperature above room temperature, the TL measurement was started $5 \mathrm{~s}$ after the excitation. This means that the natural cooling of the sample continued during the initial moments of the TL measurement but, as can be seen from Fig. 11 below, this has little to no influence on the measured glow curves.

\section{B. Optical properties}

\section{Emission and afterglow}

The emission spectrum consists of a single broad peak ( $50 \mathrm{~nm}$ FWHM) peaking around $440 \mathrm{~nm}$, in the violet region of the visible spectrum. The broadness of the peak indicates $\mathrm{Eu}^{2+}$ emission. The location and shape of the spectrum is consistent with previous reports. ${ }^{49,50,66}$

The decay of the afterglow after 5 min excitation by $365 \mathrm{~nm}$ radiation is shown in Fig. 6. During the first few minutes, the emission intensity drops rapidly, but this decrease slows down over time. As reported previously, ${ }^{64}$ the photopic intensity (not shown) drops below the $0.32 \mathrm{mcd} / \mathrm{m}^{2}$ threshold after $11 \mathrm{~h}$, but the afterglow remains visible with the unaided dark-adapted eye for at least $72 \mathrm{~h}$.

The afterglow behavior shown in Fig. 6(a) cannot be explained by assuming a single trap with first order kinetics. In that case, an exponential decay profile would be observed, following a straight line in a linear-logarithmic plot. For the case of higher order kinetics, the following profile is expected:

$$
I(t)=\frac{I_{0}}{\left(1+\frac{t}{\tau}\right)^{b /(b-1)}},
$$

where $b$ is the order of kinetics. In the limit for long time values, this should show a power-law behavior. In other words, a plot of the afterglow decay in a double-logarithmic diagram would show a straight line with a slope of $b /(1-b)$. Figure 6(b) shows that such a power-law behavior is only reached after $5 \mathrm{~h}$ or more, which makes it impossible to fit the decay profile to Eq. (3). Also, the slope of -1.28 would imply a kinetic order of $b=4.57$, a rather high value which suggests a very high importance of retrapping processes. However, such a strong influence of retrapping is not observed in TL experiments (see below). In short, the afterglow results strongly suggest the presence of a trap distribution, which will now be verified using TL experiments.

\section{Thermal quenching}

A factor which should not be overlooked when analyzing TL glow curves is the thermal quenching of the material. At higher temperatures, nonradiative decay processes become increasingly important and start dominating the radiative
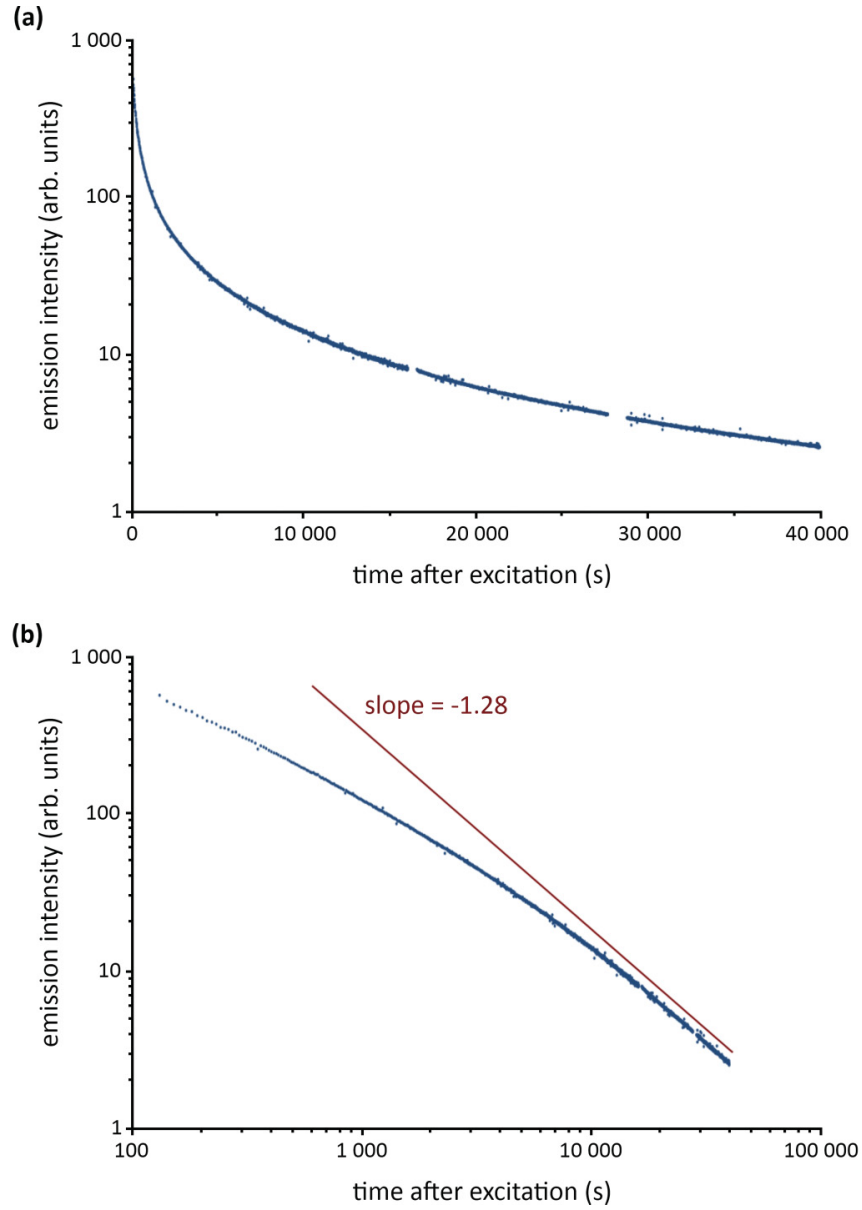

FIG. 6. (Color online) Persistent luminescence or afterglow decay of $\mathrm{CaAl}_{2} \mathrm{O}_{4}: \mathrm{Eu}, \mathrm{Nd}$ after $5 \mathrm{~min}$ excitation by $365 \mathrm{~nm}$ radiation: (a) in a linear-logarithmic diagram; (b) in a double-logarithmic diagram.

transitions. Hence, the emitted light intensity starts to drop upon increasing temperature. In the present case we are not interested in the radiative transitions only, but in the total number of charge carriers released from the traps. As previously pointed out by various authors, the glow curves measured during a TL experiment should therefore be corrected for thermal quenching in order to represent the actual number of released charge carriers. ${ }^{10,20,42}$

The thermal quenching profile for $\mathrm{CaAl}_{2} \mathrm{O}_{4}: \mathrm{Eu}, \mathrm{Nd}$ is given in Fig. 7. A fit to a standard "single-barrier" model ${ }^{67}$

$$
I(T)=\frac{I_{0}}{1+\frac{\Gamma_{0}}{\Gamma_{v}} \exp \left(\frac{-\Delta E}{k T}\right)}
$$

is used to obtain a smooth profile for correcting the TL glow curves. The quenching temperature, defined as the temperature where the emission intensity has dropped by $50 \%$, is $94{ }^{\circ} \mathrm{C}$ ( $367 \mathrm{~K})$, a rather low value compared to other luminescent materials. Since the sample in a TL experiment is typically heated to $250^{\circ} \mathrm{C}$ or higher, it is essential to take the thermal quenching into account. ${ }^{20}$ At $200^{\circ} \mathrm{C}(473 \mathrm{~K})$, the intensity has already fallen by $95 \%$. Hence, even a weak glow peak at higher temperatures can represent a considerable number of detrapping charge carriers, taking into account the large fraction of nonradiative transitions in this temperature region. 


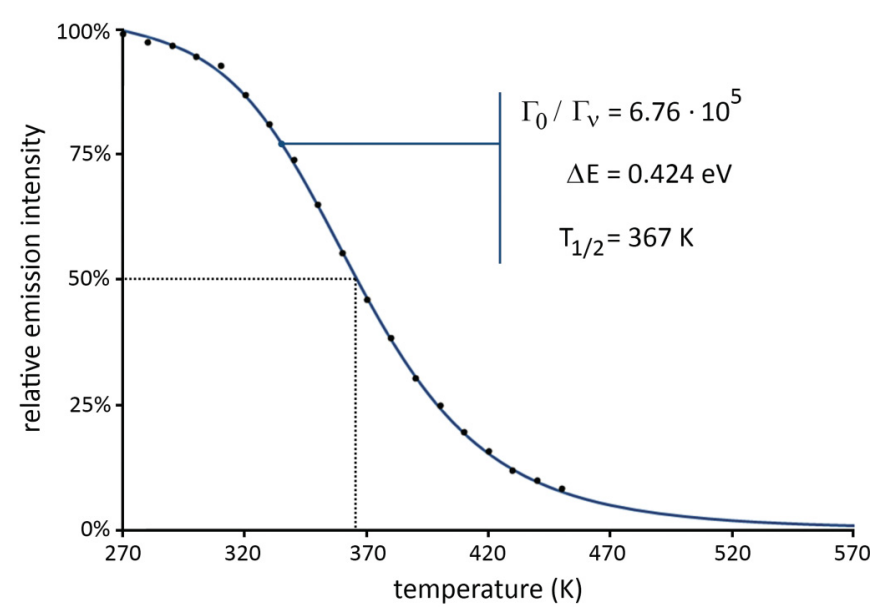

FIG. 7. (Color online) Thermal quenching for $\mathrm{CaAl}_{2} \mathrm{O}_{4}$ : $\mathrm{Eu}, \mathrm{Nd}$. Data points obtained using pulsed $337 \mathrm{~nm}$ radiation. The blue line represents the best fit to a standard single-barrier model.

All TL data in the remainder of this text have been corrected for thermal quenching.

\section{Thermoluminescence experiments}

In Fig. 8, the influence of the excitation duration on the TL glow curve of $\mathrm{CaAl}_{2} \mathrm{O}_{4}: \mathrm{Eu}, \mathrm{Nd}$ is shown. For longer durations the total amount of emitted light increases, because a larger number of traps is being filled. The location of the maximum also shows a slight shift towards lower temperatures. For $30 \mathrm{~s}$ excitation, the peak maximum is located at $483 \mathrm{~K}$, while for $360 \mathrm{~s}$ excitation this has shifted to $470 \mathrm{~K}$. This shift shows that the trap system is more complicated than a single trap obeying first order kinetics.

Compared to previous reports, ${ }^{6,41,63}$ the maximum of the glow peak is located at a rather high temperature (470 vs $420 \mathrm{~K})$. Possibly, this is due to the alternative preparation technique of the sample, and it could be an explanation for the improved afterglow lifetime of the sample compared to a

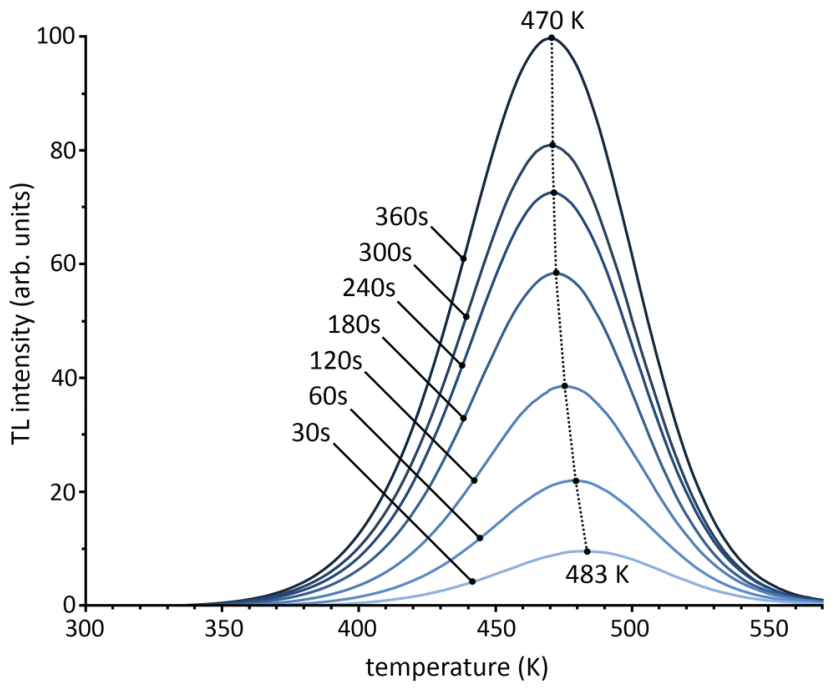

FIG. 8. (Color online) TL intensity of $\mathrm{CaAl}_{2} \mathrm{O}_{4}: \mathrm{Eu}, \mathrm{Nd}$ after 365 $\mathrm{nm}$ excitation at room temperature with various durations (30-360 s), at a heating rate of $5 \mathrm{~K} / \mathrm{s}$.

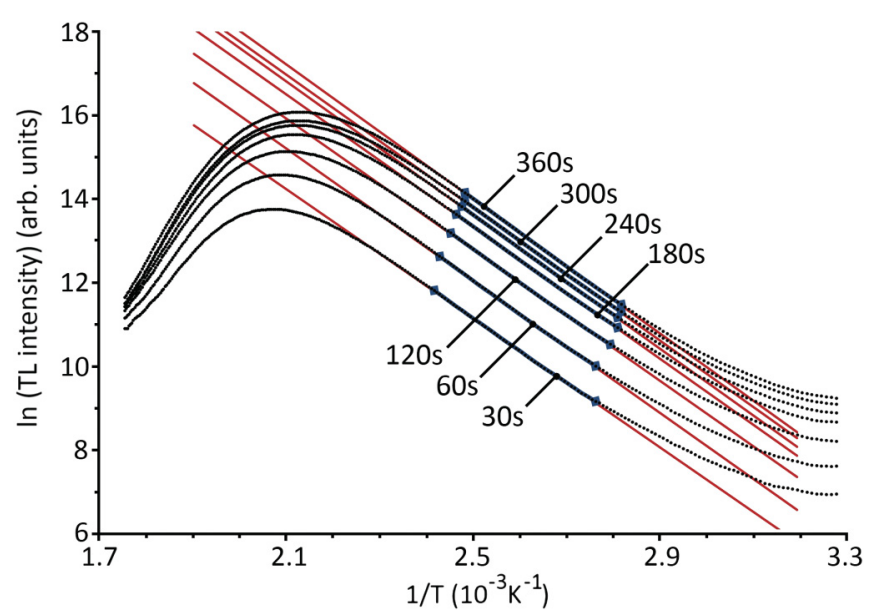

FIG. 9. (Color online) Initial rise analysis of the TL glow curves in $\mathrm{CaAl}_{2} \mathrm{O}_{4}: \mathrm{Eu}, \mathrm{Nd}$ as a function of excitation duration. The dark blue lines represent the areas of the curve used for the fitting; these are the areas where the intensity is between $1 \%$ and $15 \%$ of the maximal intensity. The red lines represent the fitting results.

benchmark $\mathrm{CaAl}_{2} \mathrm{O}_{4}: \mathrm{Eu}, \mathrm{Nd}$ persistent phosphor, as reported previously. ${ }^{64}$

Aitasalo et $a l^{41}$ found that the glow curve for $\mathrm{CaAl}_{2} \mathrm{O}_{4}$ : $\mathrm{Eu}, \mathrm{Nd}$ consists of two overlapping peaks. The main TL peak, around $350 \mathrm{~K}$, was present in all $\mathrm{CaAl}_{2} \mathrm{O}_{4}: \mathrm{Eu}, R$ ( $R=$ rare earth) samples and is therefore assumed to be related to the lattice rather than to the codopants. The peak at higher temperature is attributed to $\mathrm{Nd}^{3+}$-related traps which improve the afterglow decay time. The presence of only a single glow peak at relatively high temperature in our sample therefore indicates that the deeper Nd-related defects dominate the more shallow intrinsic traps in this sample. ${ }^{41}$ It should be noted, however, that the TL experiments in Ref. 41 were performed at low excitation intensity, which could influence the relative trap filling of both kinds of traps.

If the trap depth for each of the curves is estimated using the initial rise method, the result shown in Fig. 9 is obtained. When plotted in an Arrhenius diagram, each curve has a straight section on the low-temperature side, indicating that the assumption made for the initial rise analysis is valid. The slope of these sections, and hence the estimated trap depth, is practically independent of the excitation duration, as shown in Fig. 10. The trap depth is located between 0.66 and $0.68 \mathrm{eV}$, which is in relatively good agreement with previous estimates by Aitasalo et al. ${ }^{41}$ who found a trap at $0.67 \mathrm{eV}$, but with the additional presence of a more shallow trap around $0.55 \mathrm{eV}$ (by employing the preheating technique and the initial rise analysis).

In the remainder of this text, we will prove the presence of a continuous trap depth distribution. However, the constant trap depth as seen in Fig. 10 indicates that we are looking at the same traps in each experiment (independent of the excitation time), and that the slight shift of the glow peak maximum as seen in Fig. 8 is not caused by the presence of this distribution. Rather, the shift is most probably due to some-albeit relatively minor-retrapping.

In the case of a single discrete trap level, the expected temperature shift in the presence of retrapping can be 


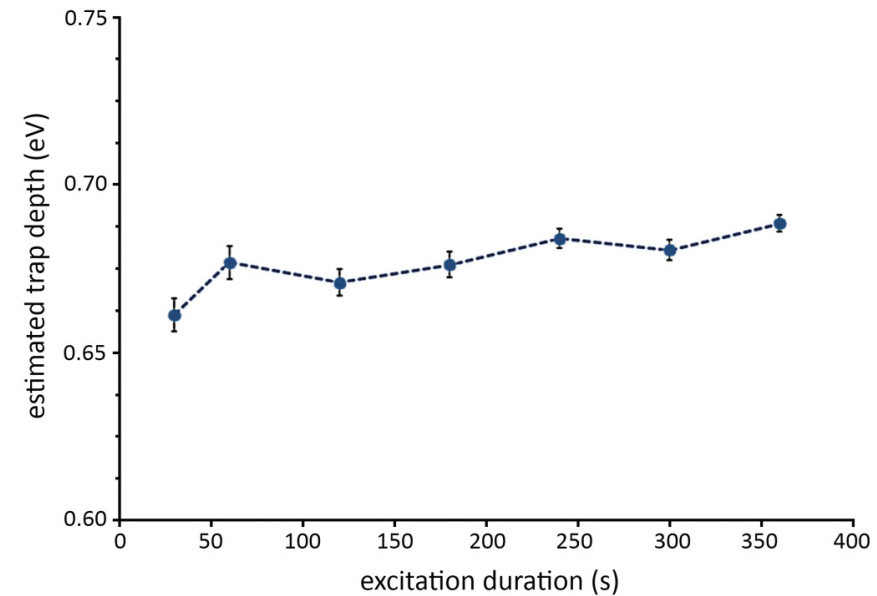

FIG. 10. (Color online) Estimated trap depth in $\mathrm{CaAl}_{2} \mathrm{O}_{4}: \mathrm{Eu}, \mathrm{Nd}$ for increasing excitation duration. The error bars indicate three times the standard deviation of the data from the fitting result.

approximated by ${ }^{20}$

$$
T_{1}-T_{2} \approx T_{1} T_{2} \frac{k(b-1)}{E_{T}} \ln f,
$$

where $T_{1}$ is the temperature of maximum intensity at a certain dose and $T_{2}$ is the temperature of maximum intensity at an $f$ times higher dose. $b$ is again the order of kinetics. Assuming Eq. (5) is also approximately valid for a continuous trap depth distribution, we can estimate the value of $b$ to be around 1.17. This indicates that some retrapping may take place.

The influence of changing the excitation temperature on the glow curves is given in Fig. 11. As expected, the total TL intensity lowers for higher excitation temperatures. Note that this also means that the trapping process is not thermally

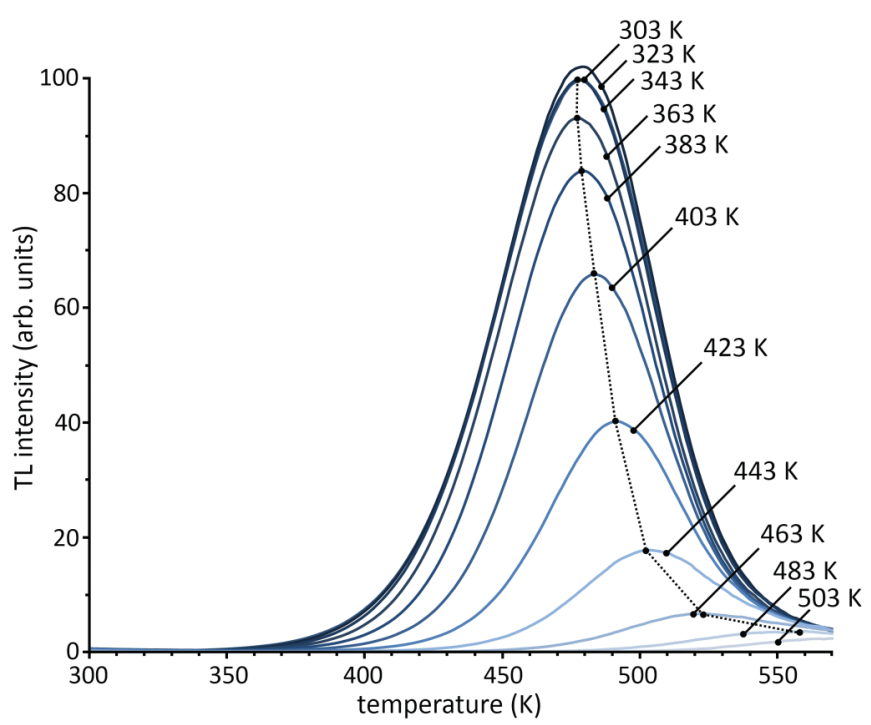

FIG. 11. (Color online) TL intensity of $\mathrm{CaAl}_{2} \mathrm{O}_{4}: \mathrm{Eu}, \mathrm{Nd}$ for various excitation temperatures, $T_{\text {exc }}$, as indicated. Samples were excited at $T_{\text {exc }}$ by $365 \mathrm{~nm}$ light for $60 \mathrm{~s}$. The TL measurement started $5 \mathrm{~s}$ after the excitation at a heating rate of $5 \mathrm{~K} / \mathrm{s}$.

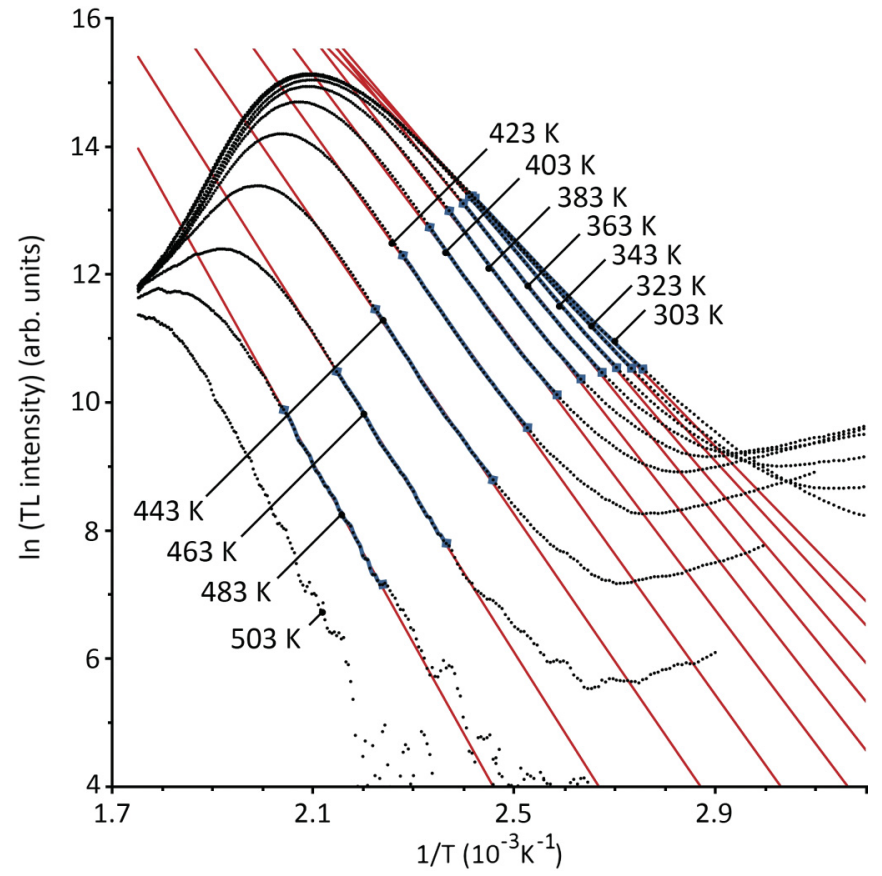

FIG. 12. (Color online) Initial rise analysis of the TL glow curves in $\mathrm{CaAl}_{2} \mathrm{O}_{4}: \mathrm{Eu}, \mathrm{Nd}$ as a function of excitation temperature. The dark blue lines represent the areas of the curve used for the fitting; these are the areas where the intensity is between $1 \%$ and $15 \%$ of the maximal intensity. The red lines represent the fitting results.

activated within the studied temperature range, as is the case for some phosphor materials, e.g., the Eu-doped nitridosilicates ${ }^{68}$ and Pr-doped lutetium pyrosilicate. ${ }^{69}$ In such phosphors, trap filling is facilitated at higher excitation temperatures, leading to more filled traps and an increase in the total TL intensity for increasing excitation temperature. This behavior is not seen in our $\mathrm{CaAl}_{2} \mathrm{O}_{4}$ : $\mathrm{Eu}$,Nd sample for the studied range of excitation temperatures.

Again, as in Fig. 8, a shift in the location of the glow peak maximum is seen, which is much larger than in the previous case (from $475 \mathrm{~K}$ at $T_{\text {exc }}=303 \mathrm{~K}$ to $560 \mathrm{~K}$ at $T_{\text {exc }}=483 \mathrm{~K}$ ), and cannot be explained by the possibility of retrapping alone. Here, the presence of a continuous trap depth distribution is strongly suggested by the data.

The initial rise analysis is shown in Fig. 12. The straight sections at the low-temperature side of the glow curves have a slope which is increasing for increasing excitation intensities. At high excitation temperatures $(503 \mathrm{~K})$ the low emission intensity makes the data too unreliable to accurately estimate the trap depth. The resulting trap depths are given in Fig. 13. Upon excitation at room temperature, the estimated depth is $0.69 \mathrm{eV}$, as in the previous experiment, but this depth increases to over $1.2 \mathrm{eV}$ for an excitation temperature of $483 \mathrm{~K}$. As discussed in Sec. II A3, the gradual deepening of the trap depth for increasing excitation temperature proves the presence of a continuous trap distribution in $\mathrm{CaAl}_{2} \mathrm{O}_{4}: \mathrm{Eu}, \mathrm{Nd}$ located roughly between 0.69 and $1.2 \mathrm{eV}$.

It must be kept in mind that the continuous trap depth distribution can extend further on both ends, i.e., it is possible that even shallower and even deeper traps exist. However, these are difficult to detect experimentally. Traps which are 


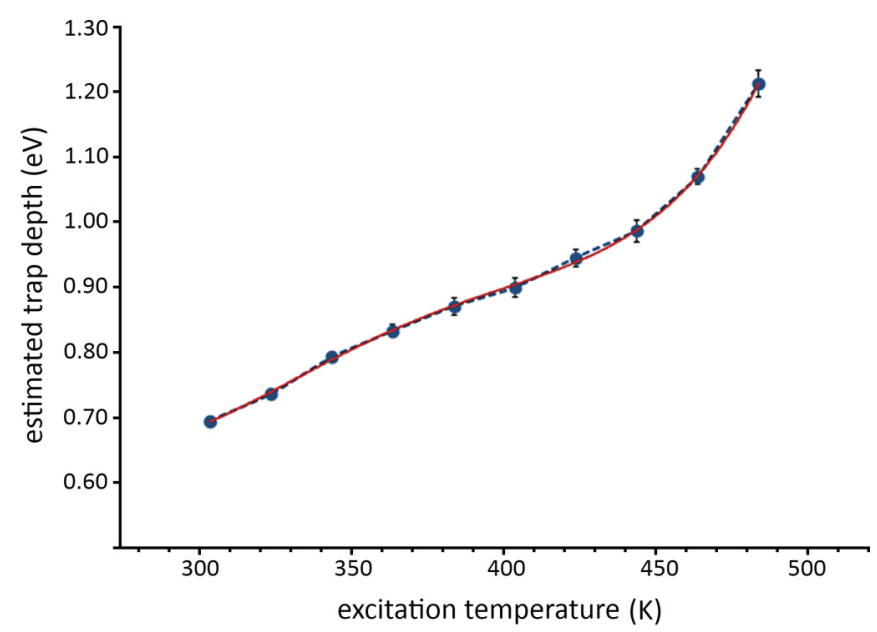

FIG. 13. (Color online) Estimated trap depth in $\mathrm{CaAl}_{2} \mathrm{O}_{4}: \mathrm{Eu}, \mathrm{Nd}$ as a function of excitation temperature. The red line is a polynomial fit through the obtained results.

too shallow could only be seen when exciting at temperatures below room temperature, which requires cooling of the sample. Not only does this require a more advanced experimental setup, but it might also be more difficult to efficiently fill traps at these lower temperatures due to thermal activation of the trapping. ${ }^{68}$

On the other hand, traps deeper than $1.2 \mathrm{eV}$ are difficult to detect because of the low intensity of the involved glow peaks for these high excitation temperatures (see Fig. 11). Also, the glow peaks in this case are located at high readout temperatures $(600-700 \mathrm{~K})$, where the influence of thermal quenching is very high. The combination of these factors makes an analysis of possible deep traps unreliable. For applications, however, these deep traps have little to no effect on the afterglow at room temperature.

\section{Shape of the trap depth distribution}

To determine the shape of the trap depth distribution, the glow curves in Fig. 11 are integrated to obtain the total TL intensity for each measurement. As described in Sec. II C, the level density can be estimated at various depths by taking the difference between each of these values. For example, when exciting at $383 \mathrm{~K}$ the calculated trap depth was $0.87 \mathrm{eV}$, while exciting at $403 \mathrm{~K}$ gave a trap depth of $0.90 \mathrm{eV}$. Hence, the difference between the integrated TL intensity of the curves at $T_{\mathrm{exc}}=383 \mathrm{~K}$ and $T_{\mathrm{exc}}=403 \mathrm{~K}$ is a direct measure for the number of traps in between 0.87 and $0.90 \mathrm{eV}$.

Figure 14 shows the final result of this analysis. A Gaussian profile was found, with a maximum around $0.9 \mathrm{eV}$, but extending from at least 0.7 to $1.2 \mathrm{eV}$. The best fit to a Gaussian profile is also shown (in red), from which we find a maximum around $0.91 \mathrm{eV}$, with a standard deviation of about $0.07 \mathrm{eV}$. As mentioned previously, the Gaussian shape of the profile is not unexpected from a statistical point of view. Furthermore, looking at the work of Medlin et al. ${ }^{31}$ the shape and broadness of the TL glow curves and the shape of the afterglow decay are both qualitatively consistent with the presence of such a Gaussian-shaped trap depth distribution.
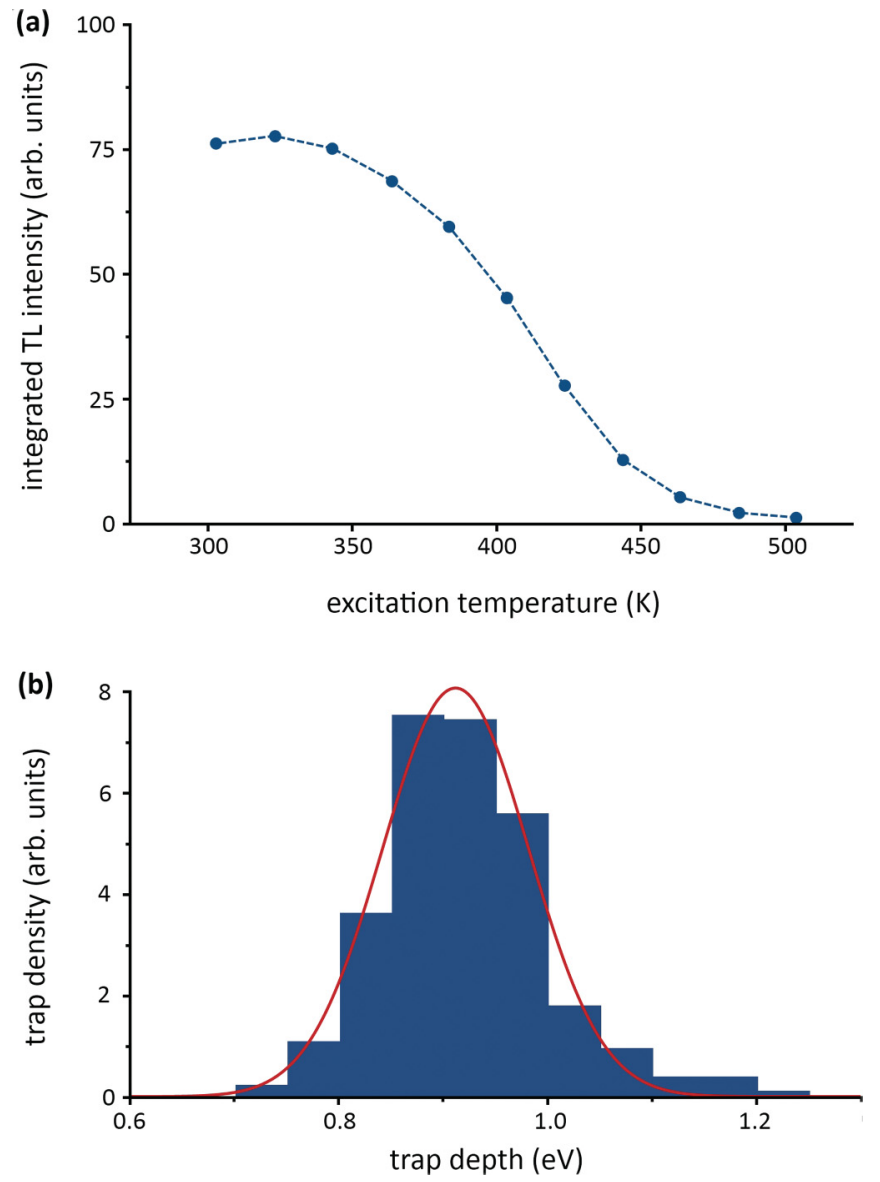

FIG. 14. (Color online) (a) Integrated TL intensity in $\mathrm{CaAl}_{2} \mathrm{O}_{4}: \mathrm{Eu}, \mathrm{Nd}$ for various excitation temperatures. (b) Density of energy levels for the trap depth distribution in $\mathrm{CaAl}_{2} \mathrm{O}_{4}: \mathrm{Eu}, \mathrm{Nd}$. The red line shows the best fit to a Gaussian profile.

\section{SUMMARY}

In summary, the following steps should be taken for such a trap depth probing:

(1) Measuring the thermal quenching of the sample in order to properly correct the thermoluminescence data.

(2) Measuring the dose dependence of the thermoluminescence glow curves to verify the presence of higher order kinetics.

(3) Measuring the thermoluminescence glow curves after exciting at various temperatures $T_{\text {exc }}$. The initial rise analysis can be performed to estimate the trap depths. An estimated trap depth which varies continuously as a function of $T_{\mathrm{exc}}$ is a strong indication for the presence of a continuous trap depth distribution.

(4) If the trapping process is not thermally activated, the area under the glow curves obtained in (3) can be used to estimate the shape of the continuous trap depth distribution. If thermal activation of the trapping is seen, the areas should first be corrected for this.

\section{CONCLUSIONS}

The activation energies in persistent phosphors can be determined in an accurate and reliable way by combining 
a series of TL experiments at different excitation duration and with different excitation temperature with the initial rise method. In this way, it is possible to reveal the presence of a continuous distribution of trap depths and to estimate the shape and depth of this distribution.

As an example, the activation energies in $\mathrm{CaAl}_{2} \mathrm{O}_{4}$ : $\mathrm{Eu}, \mathrm{Nd}$, responsible for the persistent luminescence, were studied experimentally. The energies are composed of a continuous distribution which is more or less Gaussian in shape, with the highest density of available trap depths in the region around $0.9 \mathrm{eV}$ and tails extending from at least 0.7 to $1.2 \mathrm{eV}$. The described procedure can be applied to other persistent phosphors, storage phosphors, and scintillators to obtain crucial information on the activation energies.

\section{ACKNOWLEDGMENTS}

K.V.d.E. is financially supported by the Special Research Fund (BOF) at Ghent University. Many thanks to the team at Delft University of Technology, especially Pieter Dorenbos and Jonathan de Vries, for assistance in TL experiments and for fruitful discussions. We also thank Nurşen Avcı for the sample preparation and Jonas Botterman for valuable TL discussions.
${ }^{1}$ M. Lastusaari, T. Laamanen, M. Malkamäki, K. O. Eskola, A. Kotlov, S. Carlson, E. Welter, H. F. Brito, M. Bettinelli, H. Jungner, and J. Hölsä, Eur. J. Mineral. 24, 885 (2012).

${ }^{2}$ T. Matsuzawa, Y. Aoki, N. Takeuchi, and Y. Murayama, J. Electrochem. Soc. 143, 2670 (1996).

${ }^{3}$ Y. Lin, Z. Tang, Z. Zhang, X. Wang, and J. Zhang, J. Mater. Sci. Lett. 20, 1505 (2001).

${ }^{4}$ Y. Lin, Z. Tang, and Z. Zhang, Mater. Lett. 51, 14 (2001).

${ }^{5}$ X. Wang, Z. Zhang, Z. Tang, and Y. Lin, Mater. Chem. Phys. 80, 1 (2003).

${ }^{6}$ J. Hölsä, H. Jungner, M. Lastusaari, and J. Niittykoski, J. Alloys Compd. 323-324, 326 (2001).

${ }^{7}$ K. Van den Eeckhout, P. F. Smet, and D. Poelman, Materials 3, 2536 (2010).

${ }^{8}$ K. Korthout, K. Van den Eeckhout, J. Botterman, S. Nikitenko, D. Poelman, and P. F. Smet, Phys. Rev. B 84, 085140 (2011).

${ }^{9}$ M. Fasoli, A. Vedda, M. Nikl, C. Jiang, B. P. Uberuaga, D. A. Andersson, K. J. McClellan, and C. R. Stanek, Phys. Rev. B 84, 081102(R) (2011).

${ }^{10} \mathrm{R}$. Chen and S. W. S. McKeever, Theory of Thermoluminescence and Related Phenomena (World Scientific, Singapore, 1997).

${ }^{11}$ V. Pagonis, G. Kitis, and C. Furetta, Numerical and Practical Exercises in Thermoluminescence (Springer, New York, 2006).

${ }^{12}$ W. Hoogenstraaten, Philips Res. Rep. 13, 515 (1958).

${ }^{13}$ R. Chen, J. Electrochem. Soc. 116, 1254 (1969).

${ }^{14}$ A. J. J. Bos, T. M. Piters, J. M. Gómez Ros, and A. Delgado, Radiat. Prot. Dosim. 51, 257 (1994).

${ }^{15}$ A. J. J. Bos, T. M. Piters, J. M. Gómez-Ros, and A. Delgado, Radiat. Prot. Dosim. 47, 473 (1993).

${ }^{16}$ J. T. Randall and M. H. F. Wilkins, Proc. R. Soc. London, Ser. A 184, 390 (1945).

${ }^{17}$ J. T. Randall and M. H. F. Wilkins, Proc. R. Soc. London, Ser. A 184, 365 (1945).

${ }^{18}$ G. F. J. Garlick and A. F. Gibson, Proc. Phys. Soc. 60, 574 (1948).

${ }^{19}$ C. E. May and J. A. Partridge, J. Chem. Phys. 40, 1401 (1964).

${ }^{20}$ A. J. J. Bos, Radiat. Meas. 41, S45 (2007).

${ }^{21}$ W. F. Hornyak and R. Chen, J. Lumin. 44, 73 (1989).

${ }^{22}$ W. Pan, G. Ning, X. Zhang, J. Wang, Y. Lin, and J. Ye, J. Lumin. 128, 1975 (2008).

${ }^{23}$ F. B. Dejene, D. B. Bem, and H. C. Swart, J. Rare Earths 28, 272 (2010).

${ }^{24}$ F. Gutiérrez-Martín, F. Fernández-Martinez, P. Díaz, C. Colón, and A. Alonso-Medina, J. Alloys Compd. 501, 193 (2010).
${ }^{25}$ Z.-H. Ju, S.-H. Zhang, X.-P. Gao, X.-L. Tang, and W.-S. Liu, J. Alloys Compd. 509, 8082 (2011).

${ }^{26}$ A. Bosacchi, S. Franchi, and B. Bosacchi, Phys. Rev. B 10, 5235 (1974).

${ }^{27}$ H. Gobrecht and D. Hofmann, J. Phys. Chem. Solids 27, 509 (1966).

${ }^{28}$ J. Hagekyriakou and R. J. Fleming, J. Phys. D: Appl. Phys. 15, 163 (1982).

${ }^{29}$ W. F. Hornyak, R. Chen, and A. Franklin, Phys. Rev. B 46, 8036 (1992).

${ }^{30} \mathrm{G}$. Kitis and J. M. Gomez-Ros, Nucl. Instrum. Methods Phys. Res. A 440, 224 (2000).

${ }^{31}$ W. L. Medlin, Phys. Rev. 123, 502 (1961).

${ }^{32}$ L. F. Pender and R. J. Fleming, J. Phys. C 10, 1561 (1977).

${ }^{33}$ L. F. Pender and R. J. Fleming, J. Phys. C 10, 1571 (1977).

${ }^{34}$ T. Sakurai and R. K. Gartia, J. Appl. Phys. 82, 5722 (1997).

${ }^{35}$ T. Sakurai, K. Shoji, K. Itoh, and R. K. Gartia, J. Appl. Phys. 89, 2208 (2001).

${ }^{36}$ M. Zahedifar, S. Harooni, and E. Sadeghi, Nucl. Instrum. Methods Phys. Res. A 654, 569 (2011).

${ }^{37}$ M. Zahedifar, L. Karimi, and M. J. Kavianinia, Nucl. Instrum. Methods Phys. Res. A 564, 515 (2006).

${ }^{38}$ M. Zahedifar, P. Rezaeian, and S. Harooni, Nucl. Instrum. Methods Phys. Res. B 264, 378 (2007).

${ }^{39}$ A. Bosacchi, B. Bosacchi, S. Franchi, and L. Hernandez, Solid State Commun. 13, 1805 (1973).

${ }^{40}$ A. Lorincz, M. Puma, F. J. James, and J. H. J. Crawford, J. Appl. Phys. 53, 927 (1982).

${ }^{41}$ T. Aitasalo, J. Hölsä, H. Jungner, M. Lastusaari, and J. Niittykoski, J. Phys. Chem. B 110, 4589 (2006).

${ }^{42}$ A. Vedda, M. Nikl, M. Fasoli, E. Mihokova, J. Pejchal, M. Dusek, G. Ren, C. R. Stanek, K. J. McClellan, and D. D. Byler, Phys. Rev. B 78, 195123 (2008).

${ }^{43}$ P. Avouris and T. N. Morgan, J. Chem. Phys. 74, 4347 (1981).

${ }^{44}$ G. Rudlof, J. Becherer, and H. Glaefeke, Phys. Status Solidi A 49, K121 (1978).

${ }^{45}$ P. Kivits and H. J. L. Hagebeuk, J. Lumin. 15, 1 (1977).

${ }^{46}$ S. W. S. McKeever, Thermoluminescence of Solids (Cambridge University Press, Cambridge, UK, 1985).

${ }^{47}$ J. Nahum and A. Halperin, J. Phys. Chem. Solids 24, 823 (1963).

${ }^{48}$ K. H. Nicholas and J. Woods, Br. J. Appl. Phys. 15, 783 (1964).

${ }^{49}$ G. Blasse and A. Bril, Philips Res. Rep. 23, 201 (1968).

${ }^{50}$ F. C. Palilla, A. K. Levine, and M. R. Tomkus, J. Electrochem. Soc. 115, 642 (1968). 
${ }^{51}$ C. Chang, J. Xu, L. Jiang, D. Mao, and W. Ying, Mater. Chem. Phys. 98, 509 (2006).

${ }^{52}$ Y. Lin, Z. Tang, Z. Zhang, and C. Nan, J. Eur. Ceram. Soc. 23, 175 (2003).

${ }^{53}$ W. Hörkner and H.-K. Müller-Buschbaum, J. Inorg. Nucl. Chem. 38, 983 (1976).

${ }^{54}$ T. Aitasalo, J. Hölsä, H. Jungner, M. Lastusaari, and J. Niittykoski, J. Alloys Compd. 341, 76 (2002).

${ }^{55}$ T. Aitasalo, J. Hölsä, H. Jungner, M. Lastusaari, J. Niittykoski, M. Parkkinen, and R. Valtonen, Opt. Mater. 26, 113 (2004).

${ }^{56}$ T. Nakamura, T. Matsuzawa, C. C. Rowlands, V. Beltran-Lopez, G. M. Smith, and P. C. Riedi, J. Chem. Soc., Faraday Trans. 94, 3009 (1998).

${ }^{57}$ N. Avci, K. Korthout, M. A. Newton, P. F. Smet, and D. Poelman, Opt. Mater. Express 2, 321 (2012).

${ }^{58}$ J. Hölsä, T. Laamanen, M. Lastusaari, M. Malkamäki, E. Welter, and D. A. Zajac, Spectrochim. Acta, Part B 65, 301 (2010).

${ }^{59}$ Y. Wang and L. Wang, J. Appl. Phys. 101, 053108 (2007).

${ }^{60}$ H. Yamamoto and T. Matsuzawa, J. Lumin. 72-74, 287 (1997).
${ }^{61}$ T. Aitasalo, J. Hölsä, H. Jungner, M. Lastusaari, and J. Niittykoski, J. Lumin. 94-95, 59 (2001).

${ }^{62}$ T. Aitasalo, P. Dereń, J. Hölsä, H. Jungner, M. Lastusaari, J. Niittykoski, and W. Stręk, Radiat. Meas. 38, 515 (2004).

${ }^{63}$ T. Aitasalo, P. Dereń, J. Hölsä, H. Jungner, J.-C. Krupa, M. Lastusaari, J. Legendziewicz, J. Niittykoski, and W. Stręk, J. Solid State Chem. 171, 114 (2003).

${ }^{64}$ P. F. Smet, N. Avci, K. Van den Eeckhout, and D. Poelman, Opt. Mater. Express 2, 1306 (2012).

${ }^{65}$ A. J. J. Bos, R. M. van Duijvenvoorde, E. van der Kolk, W. Drozdowski, and P. Dorenbos, J. Lumin. 131, 1465 (2011).

${ }^{66}$ T. Katsumata, T. Nabae, K. Sasajima, and T. Matsuzawa, J. Cryst. Growth 183, 361 (1998).

${ }^{67}$ P. Dorenbos, J. Phys.: Condens. Matter 17, 8103 (2005).

${ }^{68}$ P. F. Smet, K. Van den Eeckhout, A. J. J. Bos, E. van der Kolk, and P. Dorenbos, J. Lumin. 132, 682 (2012).

${ }^{69}$ M. Fasoli, A. Vedda, E. Mihokova, and M. Nikl, Phys. Rev. B 85, 085127 (2012). 